Proyecciones

Vol. 24, No 2, pp. 121-152, August 2005.

Universidad Católica del Norte

Antofagasta - Chile

\title{
INERTIAL RELATIVITY - A FUNCTIONAL ANALYSIS REVIEW
}

\author{
NORBERTO SÁINZ \\ Universidad Católica de Valparaíso, Chile \\ Received : Julio 2004. Accepted : June 2005
}

\begin{abstract}
The theory of special relativity (TSR) exhibits an unquestionable success, at the expense of an unresolved axiomatic conflict with functional analysis and operator theory, as this paper demonstrates. These mathematical disciplines -amongst the newest developments in the field-could not possibly be incorporated into the original formulation of the TSR because they were in an incipient state at the turn of the $20^{\text {th }}$ century when the TSR was being formulated, maturing only decades later.
\end{abstract}




\section{ACKNOWLEDGEMENTS.}

I wish to express my warmest gratitude for the kindness of my thesis advisor, Professor Guillermo Gonzalez, who helped me express all my ideas in a presentable way and provided me with excellent reference material. I also thank my friend and former classmate, Professor Rafael Benguria, for his insightful counselling, and Professor Juan Vrsalovic, former Vice-Rector of the Pontifical Catholic University of Valparaiso, for his encouragement to overcome the skepticisms and resistances that my work could eventually find.

I also take special pleasure in acknowledging stimulating conversations held with Professors Enrique Tirapegui and Servet Martinez, which contributed to my more precise and robust discussion to the current theories.

\section{INTRODUCTION AND PRESENTATION OUTLINE.}

\subsection{Statement of the conflict.}

Unless the theory of special relativity (TSR) is constrained to rule in a onedimensional universe, its energy-momentum $N$-vector definition [1], [2], [3] relies on the convergence of an analytical expression where a pseudometric [4], [5] is used. Several authors refer to this as the Lorentzian [6] or LorentzMinkowskian [7] metric, as opposed to Riemannian or Euclidean metrics. The term Lorentz-Minkowskian, (or simply Minkowskian) will be preferred for this specific pseudometric used in TSR, in order to make a difference with other pseudometrics of Lorentzian nature ${ }^{1}$.

Other fundamental concept definitions in relativity -like the $N$-velocity and $N$-acceleration [9]- are also based on the convergence of analytical expressions that make use of the same pseudometric. These definitions in turn provide a basis for building up on more elaborate or complex physical concepts like boosts, the Fermi-Walker differential operator, the FermiWalker transport and the Thomas precession.

On the other hand, functional analysis has established in an absolute manner that limit uniqueness can only be guaranteed when proper ${ }^{2}$ met-

\footnotetext{
1 "Lorentzian" will be the generic name given to the distance functions that are invariant upon a proper Lorentz-Poincaré transformation [8]. In this way, the LorentzMinkowskian, Schwartzchild and Robertson-Walker pseudometrics are three types of "Lorentzian pseudometrics".

${ }^{2}$ Some authors call metric what in functional analysis is called pseudometric. In this work, the term "metric" will be used when referring to the distance function that complies with all four axioms of functional analysis. "Pseudometrics" will be any distance functions that fail to comply with one or more of said axioms. "Proper metric" will be
} 
rics are used [10], [11]. Since the fundamental constructions of mathematical analysis are limit processes, according to this analytical theory the Lorentzian pseudometrics do not make of the underlying spaces where they operate, adequate frameworks for mathematical analysis.

The theory of general relativity (TGR) does not escape from this conflict, as the Einstein tensor and the Minkowski manifold emerge too from analytical formulations that rely on the convergence of expressions to a unique limit value while employing pseudometrics of Lorentzian nature [12], [13], [14].

It will be shown herein, that the conflict is not resolved by recurring to Riemannian geometry [15], [16], [17] (or to any of the $20^{\text {th }}$ century variations of differential geometry [18]) which, though positioned at the frontiers of mathematical abstraction and generality prevailing in early $20^{\text {th }}$ century, is also bound -in its analytic aspects- by the above mentioned rules.

It will also be shown that neither STA (spacetime algebra, a generalization of geometric algebra for spacetime application) nor geometric multivector calculus can ease the conflict, because functional analysis conclusions are as valid in $N$-dimensional vector spaces as they are in the $2^{N}$ dimensional multivector spaces, where these latter, newly developed disciplines rule. As Hestenes ${ }^{3}$ says [19], geometric algebra and STA are new mathematical languages (as opposed to new mathematical theories), aimed to ease the formulation of the traditional physical theories. Similarly, in [20], Hestenes acknowledges that (traditional) differentiation and integration are in no way affected by the features of the newly developed geometric multivector calculus.

These analytical requirements for a proper metric, which had not been detected neither formulated at the turn of the $20^{\text {th }}$ century when TSR and TGR were developed ${ }^{4}$, conform an axiomatic basis of functional analysis -one of the newest achievements of mathematical analysis- which experienced its greatest developments only after 1920, culminating around 1940 with the formulation of the spectral theorem [24], [25].

\footnotetext{
a term used to stress the axiom compliant nature of a metric, versus a pseudometric.

${ }^{3}$ David O. Hestenes, Ph.D.. Research Professor of Physics, Arizona State University. Author of several papers on geometric algebra, spacetime algebra and geometric calculus.

${ }^{4}$ According to [21], the normed space axioms first appear in F. Riesz' work on compact operators in 1918 [22]. The first abstract treatment of the subject is in Banach's 1920 thesis [23].
} 


\subsection{One probable scenario for conflict development.}

One question immediately comes to mind:

If the above statements are correct, how could the scientific community of the $20^{\text {th }}$ century overlook the physical implications of this new analytical theory?

A complete and formal answer to this very pertinent question is beyond the scope of this work, and should probably belong to the field of epistemology.

Notwithstanding - at the risk of being considered overly simplistic- one probable scenario is hereby offered, because the absence of historical scenarios suitable for the development of the claimed conflict would render such conflict unfeasible.

Firstly, note that TSR was developed before 1905 and TGR before 1916. Shortly after their publication, respected observers reported experimental tests confirming -beyond doubt- Einstein's theories. The confirmed theories defied common sense at the time, amazing the physics community and astonishing the scientific world. An unexplored and apparently limitless wealth of implications into other fields of physics boosted theoretical and experimental activity.

While the physics community was overwhelmed by the unprecedented success and unforeseen implications of the new relativistic theories, mathematicians were busy migrating their theories from Riemannian geometry with Ricci calculus, to H. Weyl's (1918) and later to Cartan's (1923-24) connections. (In particular, Cartan's work on Klein spaces, replacing the general linear group by a Lie group was developed coetaneously with TGR).

Figure 1 belongs to the website of Patrick Reany at www.asu.edu, and enables a partial and complementary chronological view of the development of mathematical analysis. 


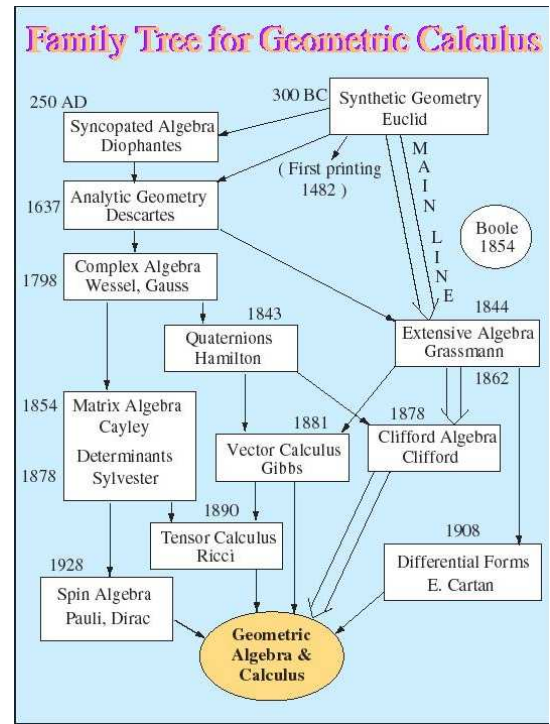

Figure 1 : Development of Geometric Calculus - Cronology

Topology and functional analysis didn't exist at that time, as they were born around 1920 with the works of Riesz and Banach. By the time these disciplines had grown to a mature theory, capable of distilling contribution to other fields of knowledge, $(1945-1950)$, relativity had gained such recognition that no newcomer, low profile and inopportune discipline like functional analysis could possibly present a conflicting threat to it.

Acknowledgely, the last sentence above is rather provocative; however, there are signs of its veracity. The following reflection is offered as evidence:

Though early $20^{\text {th }}$ century Riemannian geometry -on which TGR was based- did not require manifolds to be Hausdorff ${ }^{5}$ (the concept did not exist at the time), today every respectable treatise on differential geometry incorporates the Hausdorff requirement right from the beginning.

Inconceivably, as of today this requirement has not distilled to $\mathrm{TGR}^{6}$

\footnotetext{
26].

${ }^{5} \mathrm{~A}$ topological space where sequential limits are unique is called a Hausdorff space

${ }^{6}$ The main activities in differential geometry at the turn of the $20^{\text {th }}$ century were centered on the geometry in the Euclidean space, following the tradition of Euler and Monge. It was then difficult (and probably unnecessary) for geometers to free themselves from an absolute ambient space, usually Euclidean. See also [18], [27].

In such a scenario, derivatives are linear as a matter of course, and the chain rule is always applicable to the derivation of composite functions. There was no need to invent special axioms for these features.

In fact, Riemann surfaces were initially not subject to any topological restrictions other than orientability and countability. Riemann and the early contributors to Riemannian geometry (Schouten, Levi-Civita, Eisenhart) did not know -but at the same time did not
} 
and as a consequence, analytical expressions on the Minkowski manifold or any other pseudo-Riemannian manifolds (none of which are Hausdorff) are deemed to be correct, when in fact they are not, as will be shown in §2.4.2.

\subsection{Strategies for overcoming the difficulties.}

Some analytical difficulties with TSR and TGR have been acknowledged though, motivating the scientific community to attempt several ways of providing the relativistic theories with a suitable framework for analysis. In order to offer mathematical proof supporting the above statements of historical nature, these main attempts to develop a suitable analytical framework will now be classified into four courses of action or strategies. Later, in $\S 2.4$ hereof they will be discussed in detail:

1. Those that restrict the scope of the physical model (and therefore, of the "physically allowable" spacetime trajectories) to the future and past $N$-dimensional lightcones relative to an observer [13], [28].

The early approaches -many of which used this strategy- incorrectly assume that, by ensuring (for material objects, and within the lightcones) that $\sqrt{1-\beta^{2}} \neq 0[13]$, analytical expressions would therein be well defined.

2. Those that further restrict the validity of the analytical expressions contained in TSR and TGR, so they rule exclusively along "physically allowable" (see point 1 above) inertial spacetime trajectories, and then attempt to combine $N$ of these one-dimensional analyses made along linearly independent inertial trajectories, to span an $N$ dimensional spacetime [13], [29], [30]. Pseudo-Riemannian geometry and STA (with its generalization for allowing a non-positive-definite Clifford multivector product) fall into this category.

Though Lorentzian distance functions truly qualify as proper metrics in each of these one-dimensional spaces, the incorrect assumption here is that, in pseudometric spaces, directional derivatives would still maintain their linear features with respect to the direction of

need to explicitly impose- the Hausdorff requirement or positive-definiteness axiom that we see in today's treatises on Riemannian geometry.

Other contributors to non-Riemannian forms of differential geometry (E. Cartan, and particularly those of early $20^{\text {th }}$ century) also ignored these axioms. Unluckily, some of their contributions allowed (or even depended on) the violation of these-yet undiscovered but no less mandatory- analytical axioms. The consequences are yet to be known. 
analysis, thus allowing the linear combination of two or more onedimensional segment differentials $d s_{k}^{2}=\left(1-\beta_{k}^{2}\right) \cdot d t_{k}^{2}, k=1 \ldots N$, legitimately obtained by mathematical analysis along several given paths, into $d s^{2}=g_{\mu \nu} d x^{\mu} d x^{\nu}$, an expression that is not analytically valid in $R^{N}$ if $g_{\mu \nu}$ is required to exhibit a "Lorentzian signature" as required by STA or by Minkowski and other pseudo-Riemannian manifolds.

3. Those that extend TSR and TGR to a spacetime built on a complex underlying field.

Amongst these is an early approach [33] by Minkowski, which has been disapproved by several authors with arguments that highlight the difference existing between spacetime's Lorentzian topology and the Euclidean topology, difference considered to be one of the hallmarks of TSR and TGR, and which (these authors believe) should always be kept on sight ${ }^{8}$. Other approaches are STA and Einstein's attempt to develop a unified field theory by means of a four-dimensional complex space with Hermitian structure.

The error of these elaborations lies in an improper migration of the metric space from the real numbers $R^{N}$ to the complex numbers $C^{N} .^{9}$

4. Those that postulate the existence of "more general" mathematical theories, either having or not the intention to find an analytical framework that would allow the use of pseudometric spaces for mathematical analysis [18], [34]. As will be seen, all mathematical theories known today consistently and coherently declare this fourth course of action unfeasible.

This work will elaborate on the four strategies presented above and on the comments briefed for each of them, demonstrating that none of

\footnotetext{
7 "Lorentzian signature" is the name given by some authors [13], [31], [32] to the term-sign combination $(-+++$, or +++-$)$ appearing in the arithmetic expression for the squared-distance-value in Lorentzian pseudometrics. It differs from the Euclidean signature, in that all terms of the latter are positive.

${ }^{8}$ Misner et al. [7], comment on the inconvenience of using $x^{0}=\mathrm{i}$, , since it gives the Lorentzian segment expression $(\Delta s)^{2}=\left(\Delta x^{1}\right)^{2}+\left(\Delta x^{2}\right)^{2}+\left(\Delta x^{3}\right)^{2}+\left(\Delta x^{4}\right)^{2}$ an unacceptably Euclidean flavor, hiding "the completely different metric structure of ++ ++ geometry and -+++ geometry"... "This structure makes the machinery of the physical world function as it does.". Taylor et al. [33] comment similarly.

${ }^{9}$ The topological axioms for complex underlying spaces were also developed years after the formulation of TSR and TGR.
} 
these attempts has proved successful in achieving adequate frameworks for mathematical analysis. None of these courses of action has succeeded in guaranteeing the existence of unique limits for convergent sequences in the spaces so built. Moreover, this is hereby shown to be an impossible feat.

\subsection{Verdict generality and coherence.}

But the analysis offered herein is not restricted to a case-by-case demonstration for each highlighted strategy:

This work shows, in an absolutely general manner, that the claimed conflict indeed exists and is so far unresolved, and furthermore explains how the three mathematical theories of convergence known so far are coherent with this conclusion.

The reason is obvious. If unique limits could be guaranteed in pseudometric spaces, then the axiomatic basis of functional analysis would not be absolute - as it actually is - but relative.

This is, the axiomatic basis for mathematical analysis would be subject to an arbitrarily defined scope of validity of the model, as in 1 above; or to the manner in which the algebraic framework is built, as in 2 ; or to the nature of the underlying field, as in 3 , or to mere well-intended attempts for generalization, as in 4. Functional analysis would not exist. Not as it is known today.

\subsection{Proposal of a solution and conclusions.}

So, a fifth reconciliation strategy is hereby proposed:

1. Looking for a proper metric that would enable (under the known mathematical theories) the formulation of the desired analytical model

For as long as functional analysis and operator theory stand valid, this fifth course of action is the only way to proceed. But it has a cost: by using a metric different to that employed by TSR and TGR, some features of the traditional relativistic theories may have to be given up.

The question is then: which of the metrics suitable ${ }^{10}$ for mathematical analysis in spacetime discards the smallest and least relevant set of features of TSR and TGR?

\footnotetext{
${ }^{10}$ Metrics are optional. Selecting the appropriate one is a matter of model development strategy.
} 
This work prepares the way for answering such a question by anticipating two features of TSR and TGR that must be inevitably given up, no matter which proper metric is finally selected:

- TSR's and TGR's non-positive-definite (Lorentzian) pseudometrics. A feature highlighted, amongst others, in: [13], [32], [33], [36], [37].

- TSR and TGR's Lorentzian spacetime topology [38], [39], whose difference with Euclidean/Riemannian topologies has been repeatedly highlighted by several authors ${ }^{8}$.

\section{DEMONSTRATION.}

This demonstration is organized in two main parts:

\subsection{First part}

The first part introduces the mathematical concepts to be used. In so doing, it establishes the following facts:

1. That, according to the theory of sequential convergence, a pseudometric space is not a suitable framework for mathematical analysis. This, irrespective of the nature of the space, which can be one-dimensional, $N$-dimensional, real, complex, either a Klein, Minkowski, Riemann or pseudo-Riemannian manifold, either made out of loose or bundled fibers, which twist and twirl according to simple or complex development rules or connections. Even if the underlying space is a multivector space of dimension $2^{N}$ as lately used by STA. Even if geometric multivector calculus is used.

2. That there are two other known theories of convergence, and none of them enables building an analytical framework on pseudometric spaces. Same as above, this result is irrespective of the nature of the space, or of the way in which it is built.

3. That, if some topological space is to be the result of applying a proper metric to an underlying space (given the unsuitability of pseudometrics for the intended purposes, declared in 1 and 2 above), then this topological space must meet certain minimal conditions. 
Though this first part should be sufficient to demonstrate this work's contention, due to the controversial nature of the claims, the generalized adhesion that the challenged theories exhibit and the dramatic impact that this work would bring on them if deemed correct, additional and individual demonstrations for each of the strategies identified in $\S 1.3$ will be offered.

\subsection{Second part}

The second part of this demonstration applies the concepts introduced in the first part, and answers the questions posed in $\S 1.3$, i.e.:

1. Whether the Lorentzian pseudometrics, (which generate a pseudometric space in $R^{N}$ ) would generate a topological space apt for mathematical analysis when the scope of the physical model (and the zone of analysis) is constrained to the past and future lightcones relative to an observer.

2. Alternatively:

a) Whether mathematical analysis may or may not be made by applying the Lorentzian metrics to specific and predetermined spacetime paths contained in the above mentioned lightcones, and

b) Whether linear combinations of two or more analytical expressions of the kind obtained in 2 above (as used in the Minkowski and other pseudo-Riemannian manifolds, STA and geometric calculus) are meaningful.

3. Whether extending TSR and TGR to a spacetime built on a complex underlying field validates its analytical expressions.

4. Whether "more general" analytical theories exist that, -in flagrant opposition to functional analysis- would allow building an adequate framework for mathematical analysis in spacetimes with Lorentzian topologies.

\subsection{First part of the demonstration.}

\subsubsection{Demonstration of fact 1 in $\S 2.1$.}

As said, the fundamental constructions of mathematical analysis are limit proc- esses. Derivation and integration rely on the convergence of the math- 
ematical process to a limit value. In order to substitute equals for equals, this limit value must be unique [40].

A topological space where sequential limits are unique is called a Hausdorff space [26].

A topological space is a Hausdorff space (iff) distinct elements in such space exhibit disjoint neighborhoods [26].

It follows from the above that no pseudometric space is a Hausdorff space. In effect: if $X$ is a pseudometric space, then there exist two distinct elements $\mathbf{O}$ and $\mathbf{A}$ in that space, such that their distance is null. This means that $\mathbf{A}$ is in every neighborhood of $\mathbf{O}$ and vice versa. Consequently, the intersection of any neighborhood of $\mathbf{O}$ with any neighborhood of $\mathbf{A}$ contains at least these two elements: $\mathbf{O}$ and $\mathbf{A}$. Though $\mathbf{O}$ and $\mathbf{A}$ are distinct elements in $X$, no disjoint neighborhoods of $\mathbf{O}$ and $\mathbf{A}$ exist.

Note that no restrictions have been imposed on $X$, therefore the demonstration above is valid for any pseudometric space $X$, irrespective of whether it is one-dimensional, $N$-dimensional, $2^{N}$-dimensional, real, complex, either a Klein Minkowski, Riemann or pseudo-Riemannian manifold, $N$ dimensional vector, or $2^{N}$-dimensional multivector space, etc.

Sequential limits are not unique in pseudometric spaces.

The harshness and generality of the above conclusion are overwhelming ${ }^{11}$. Since no pseudometric space is a Hausdorff space, sequences are not guaranteed therein to converge to limits that are unique. In these spaces, equals cannot be substituted for equals and therefore the fundamental processes of mathematical analysis cannot be uniquely defined.

The above conclusion should be enough to invalidate all analytical constructions in the traditional spacetime. For example, according to the above, Sir Roger Penrose's" definition [42] of spacetime as: "a real, fourdimensional connected smooth Hausdorff manifold on which is defined a global smooth nondegenerate Lorentzian metric", is an oxymoron ${ }^{13}$.

Because the above conclusion is an essential part of this work, it is important to highlight once again that in its derivation, no use of (or reference

\footnotetext{
${ }^{11}$ Readers still believing that Minkowski and other pseudo-Riemannian manifolds can save the issue are kindly invited to refer to section $\S 2.4 .2$

${ }^{12}$ Sir Roger Penrose, Ph.D., born in Colchester, England, 1931, is the Rouse Ball Professor of Mathematics at the University of Oxford in 1973, a post he holds to this day. He has made significant contributions to mathematical physics. (C) Malcolm Macgregor - Roger Penrose Biography 14 Nov 2004).

${ }^{13}$ Oxymoron: A figure of speech in which opposite or contradictory ideas or terms are combined. Webster's New World Dictionary, $3^{\text {rd }}$ College Edition, 1988.
} 
to) any other properties or features of the space $X$ have been made (except that it is a pseudometric space).

\subsubsection{Demonstration of fact 2 in $\S 2.1$.}

Is there any other mathematical theory to which pseudometrics can turn, in the hope of an alternative byway to the above verdict?

Three theories of convergence are known to-date:

- The theory of sequential convergence

- The net theory of convergence (Moore-Smith)

- The filter theory of convergence $\left(\operatorname{Cartan}^{14}\right)$

As already seen, the theory of sequential convergence declares that in pseudometric spaces there is no guarantee of limit uniqueness for convergent sequences. The net theory has been proved equivalent to the sequential theory of convergence [41].

No contradictions have been found between these two theories and the filter theory. Their equivalence is highly suspected, though it has not been proved yet [43].

In any case, it is clear that the filter theory does not endorse limit uniqueness in pseudometric spaces, as this would constitute an essential and extremely conspicuous difference respect the other two theories, giving way for a conclusive negative declaration in respect of their suspected equivalence.

This demonstrates fact 2. Again, this conclusion is absolutely general and independent of any other properties or features of the underlying spaces in question.

\subsubsection{Demonstration of fact 3 in $\S 2.1$.}

A topological space that can be generated by the application of a metric to a linear space is called metrizable.

A topological space is metrizable (iff) it is pseudometrizable and it is a $\mathrm{T}_{1}$ - space $^{15}[45]$.

\footnotetext{
${ }^{14}$ Henri Cartan, known for his works on analytic functions, theory of sheaves, homological, algebraic and potential theories. Not to be confused with his father, Élie Cartan (1869 - 1951), also a distinguished mathematician.

${ }^{15} \mathrm{~A}$ topological space is a $\mathrm{T}_{1}$-space (iff) each set consisting of a single point is closed [44].
} 
These are the minimal conditions to be met by any topological space resultant of the metrization of an underlying space, which demonstrates fact 3 .

\subsection{Second part of the demonstration.}

As anticipated in $\S 2$, the overwhelmingly harsh and general nature of the conclusions reached under $\S 2.3$ make specific (or personalized) demonstrations unnecessary for all kinds of strategies aimed to perform mathematical analysis in spacetime while preserving its traditional topology.

Notwithstanding, considering the generalized adhesion to many of these strategies -and the dramatic impact that this work would bring on them if deemed correct- specific demonstrations will be offered for each.

\subsubsection{Answering question 1 of 2.2 .}

Even if TSR is restricted to the subspace limited by the future and past lightcones relative to an observer, (see Figure 2 for an $R^{2}$ representation) the mathematical conflict presented in 1 and 2 of $\S 2.1$ is not resolved.

This conflict invalidates mathematical analyses made in the interior of the cones, because it prevents the convergence to a unique limit value, of expressions such as:

$$
\mathbf{E}=m \frac{d \mathbf{r}(\tau)}{d \tau}=m \lim _{\Delta \tau \rightarrow 0} \frac{\mathbf{r}(\tau+\Delta \tau)-\mathbf{r}(\tau)}{\Delta \tau}
$$

where

$$
\mathbf{r} \in R^{4}, \mathbf{r} \equiv\left(r_{1}, \ldots, r_{4}\right), \text { and } \Delta \tau(\Delta \mathbf{r}) \equiv \sqrt{\left(\Delta r_{1}\right)^{2}-\sum_{i=2}^{4}\left(\Delta r_{i}\right)^{2}}
$$

Other ways of writing (2.2) may be found:

- in the inertial theory, where paths are straight, $\Delta \mathbf{r} \equiv \mathbf{b}-\mathbf{a}$ and $\mathrm{d}_{L}(\mathbf{a}, \mathbf{b})$ is the Lorentzian distance:

$$
\Delta \tau(\mathbf{b}-\mathbf{a}) \equiv \mathrm{d}_{L}(\mathbf{a}, \mathbf{b}) \equiv \sqrt{\left(b_{1}-a_{1}\right)^{2}-\sum_{i=2}^{4}\left(b_{i}-a_{i}\right)^{2}}
$$

- In the general theory, where paths are curved and infinitesimals must be used: 


$$
\Delta \tau \equiv \int d \tau, \text { where } d \tau \equiv \sqrt{\left(d r_{1}\right)^{2}-\sum_{i=2}^{4}\left(d r_{i}\right)^{2}}
$$

If (2.1) is to be valid in all of $R^{N}$ (the case when (2.1) is restricted to one-dimensional paths in $R^{N}$ is treated in subsection $\S 2.4 .2$ hereof), then each component of $\Delta \mathbf{r}$ (i.e., $\left.\Delta \mathrm{r}_{i}, i=1, \ldots 4\right)$, must be allowed to vary independently from one another when varying $\Delta \tau$. Similarly when $\mathrm{d} \tau$ is used.

The mathematical conflict in question is not resolved because, in spite of the conic restrictions, the subspace so defined is not Hausdorff (unless the underlying topological space is one-dimensional, as will be seen in section $\S 2.4 .2$ below). Every neighborhood of every point intersects every neighborhood of every other point in the space so restricted.

The demonstration of the above statement will be elaborated in two stages. The first stage will deal with Lorentzian neighborhoods which include the interior of the lightcones as well as their boundary. The second stage will deal with neighborhoods that include only the interior of the lightcones.

First stage: consider first $R^{2}$ where the Lorentzian distance function (2.3) is applied (see Figure 2).

Let $\mathbf{Q}$ be any point of $R^{2}$, and define the lightcone relative to $\mathbf{Q}, \bar{L}(\mathbf{Q})$, as:

$$
\bar{L}(\mathbf{Q})=\left\{\mathbf{r} \in R^{2} \ni \mathrm{d}_{L}^{2}(\mathbf{Q}, \mathbf{r}) \geq 0\right\}
$$

For sake of clarity, define the interior of $\bar{L}(\mathbf{Q})$ as:

$$
L(\mathbf{Q})=\left\{\mathbf{r} \in R^{2} \ni \mathrm{d}_{L}^{2}(\mathbf{Q}, \mathbf{r})>0\right\}
$$

and the boundary or border of $\bar{L}(\mathbf{Q})$ as:

$$
\dot{L}(\mathbf{Q})=\left\{\mathbf{r} \in R^{2} \ni \mathrm{d}_{L}^{2}(\mathbf{Q}, \mathbf{r})=0\right\}
$$

It happens that:

$$
\begin{aligned}
\bar{L}(\mathbf{Q}) & =L(\mathbf{Q}) \cup \dot{L}(\mathbf{Q}) \\
\dot{L}(\mathbf{Q}) & =\bar{L}(\mathbf{Q}) \sim L(\mathbf{Q})
\end{aligned}
$$

Define the future lightcone relative to $\mathbf{Q}$, as:

$$
\bar{L}^{+}(\mathbf{Q})=\bar{L}(\mathbf{Q}) \cap\left\{\mathbf{r} \in R^{2} \ni r_{1} \geq q_{1}\right\}
$$


Use a procedure equivalent to that applied in (2.10) for defining the interior and boundary of this future lightcone similarly.

Apply (2.5) through (2.10) to the origin $\mathbf{O}$ of the coordinate system to form a new underlying space, by restricting $R^{2}$ to $\bar{L}^{+}(\mathbf{O})$, as in Figure 2.

Select now any other point, $\mathbf{A}$, in $\bar{L}^{+}(\mathbf{O})$ and consider the past lightcone relative to it, $\bar{L}^{-}(\mathbf{A})$ :

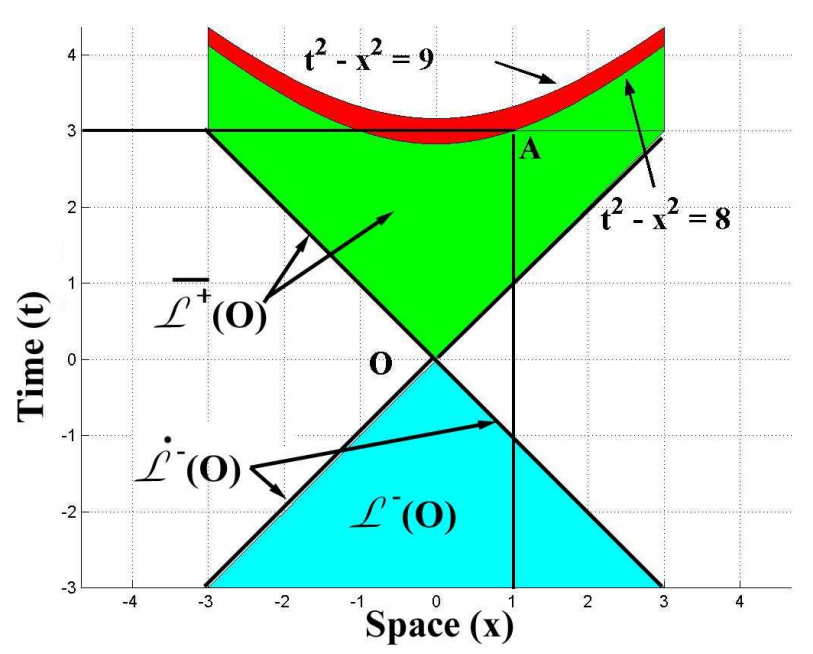

Figure 2 : Lightcones emerging from $\mathrm{O}$ in spacetime

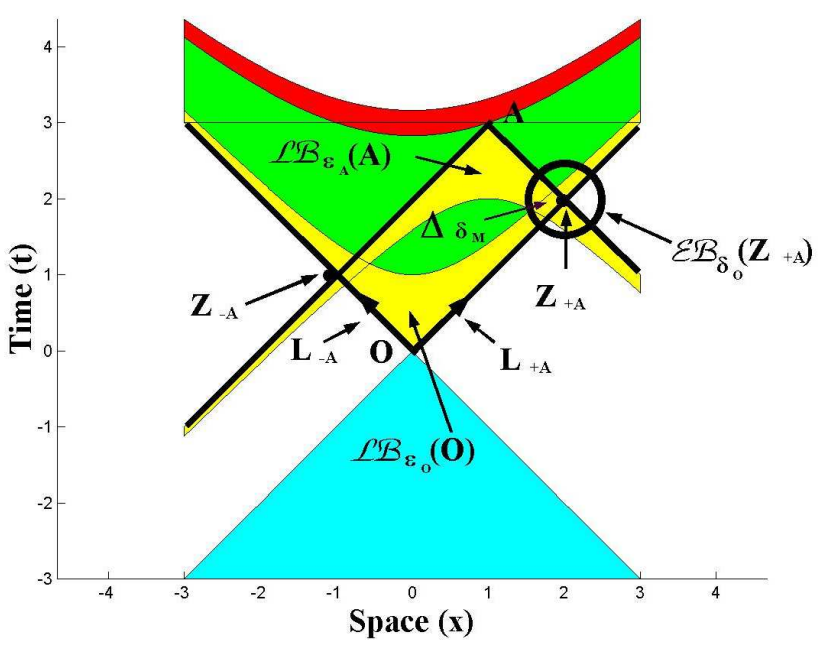

Figure 3 : Intersection of lightcones emerging from $\mathrm{O}$ and $\mathrm{A}$ 
Figure 3 schematizes this construction.

Define the interior and boundary of this new lightcone, past to $\mathbf{A}$, in a manner similar to (2.11) above.

Then, at least two ${ }^{16}$ spacetime points, $\mathbf{Z}_{+} \mathbf{A}$ and $\mathbf{Z}_{-\mathbf{A}}$ (individually referred to either of them in a generic way as $\mathbf{Z}_{ \pm \mathbf{A}}$ ), may be found in the intersection of both boundaries, $\dot{L}^{+}(\mathbf{O}) \cap \dot{L}^{-}(\mathbf{A})$, separated from $\mathbf{O}$ and $\mathbf{A}$ by a null Lorentzian distance (see Figure 3, for reference):

$$
\mathbf{Z}_{ \pm \mathbf{A}}=\left\langle\mathbf{A} \mid \mathbf{L}_{ \pm \mathbf{A}}\right\rangle \mathbf{L}_{ \pm \mathbf{A}}
$$

where:

- $\mathbf{L}_{+\mathbf{A}}$ is an event future to $\mathbf{O}$, separated from it by a unit temporal lapse and a unit spatial distance towards $\mathbf{A}$.

- $\mathbf{L}_{-\mathbf{A}}$ is an event future to $\mathbf{O}$, separated from it by a unit temporal lapse and a unit spatial distance away from $\mathbf{A}$.

- $\mathbf{L}_{ \pm \mathbf{A}}$ uses the same notational convention as $\mathbf{Z}_{ \pm \mathbf{A}}$.

- If $\mathbf{A}$ is not separated from $\mathbf{O}$ by a finite spatial distance, then let $\mathbf{L}_{ \pm \mathbf{A}}$ be any two events separated from $\mathbf{O}$ by a unit temporal lapse and a unitary spatial distance in any two linearly independent directions.

- $\langle\cdot \mid \cdot\rangle$ stands for the inner product operator.

Each of these points $\mathbf{Z}_{ \pm \mathbf{A}}$ is common to every local neighborhood of $\mathbf{A}$ and $\mathbf{O}$. Since $\mathbf{O}$ and $\mathbf{A}$ are arbitrary, it follows that no distinct points in the subspace so defined exhibit disjoint neighborhoods.

Second stage: the conflict under study is maintained even if the scope of the model excludes the conic boundaries or surfaces, $\dot{L}^{+}(\mathbf{O})$ and $\dot{L}^{-}(\mathbf{A})$ (this is, even if null geodesics are considered a physically unreachable limit, and are excluded from the scope of the model). This is so because, again, in spite of the increased severity of the new conic restrictions, the subspace so defined is still not Hausdorff.

In effect: Consider $\mathbf{O}$ and $\mathbf{A}$ given as before, with arbitrary Lorentzian neighborhoods generated by $\mathrm{d}_{L}$ as defined in (2.3), plus the more stringent

\footnotetext{
${ }^{16}$ The number of points depends on the dimension of $R^{N}$. If $N=2$, then two such points exist. If $N>2$, an infinite number of points exists.
} 
conic restrictions. It will be shown that there is still some point in the space so constrained, belonging to both neighborhoods.

For this, think of $\mathrm{d}_{L}$ as a function mapping $R^{2} \times R^{2} \mapsto R$. Use the Euclidean metric $\mathrm{d}_{E}$ in both spaces. Then,

$$
\mathrm{d}_{L}(\cdot, \cdot):\left(R^{2} \times R^{2}, \mathrm{~d}_{E}\right) \rightarrow\left(R, \mathrm{~d}_{E}\right)
$$

The Lorentzian distance function (2.13) is symmetric and continuous with the Euclidean metric in $R^{4}$ and $R$.

In addition, because the Euclidean metric is used, every point lying on the boundary of the lightcones (future to $\mathbf{O}$ as well as past to $\mathbf{A}$ ) is an adherence point of the interior of the respective cone. This means that each of the points $\mathbf{Z}_{ \pm \mathbf{A}}$, lying simultaneously on the boundaries of both lightcones, is an adherence point of the interior of their intersection.

Given any two Lorentzian neighborhoods, one for $\mathbf{O}$ and another for $\mathbf{A}$, Lorentzian balls of a certain diameter $\varepsilon_{\mathbf{O}}$ and $\varepsilon_{\mathbf{A}}$, may always be found, respectively contained in each. Call them $L B_{\varepsilon_{\mathbf{O}}}(\mathbf{O})$ and $L B_{\varepsilon_{\mathbf{A}}}(\mathbf{A})$ (see Figure 3):

$$
\begin{aligned}
& L B_{\varepsilon_{\mathbf{O}}}(\mathbf{O})=\left\{\mathbf{r} \ni\left|\mathrm{d}_{L}(\mathbf{O}, \mathbf{r})\right|<\varepsilon_{\mathbf{O}}\right\} \\
& L B_{\varepsilon_{\mathbf{A}}}(\mathbf{A})=\left\{\mathbf{r} \ni\left|\mathrm{d}_{L}(\mathbf{A}, \mathbf{r})\right|<\varepsilon_{\mathbf{A}}\right\}
\end{aligned}
$$

The Euclidean continuity of $\mathrm{d}_{L}$ in $R^{4}$ implies that:

$$
\begin{aligned}
& \forall \varepsilon_{\mathbf{O}} \exists \delta_{\mathbf{O}} \ni \forall \mathbf{r} \in E B_{\delta_{\mathbf{O}}}\left(\mathbf{Z}_{ \pm \mathbf{A}}\right) \rightarrow\left|\mathrm{d}_{L}(\mathbf{O}, \mathbf{r})\right|<\varepsilon_{\mathbf{O}} \\
& \forall \varepsilon_{\mathbf{A}} \exists \delta_{\mathbf{A}} \ni \forall \mathbf{r} \in E B_{\delta_{\mathbf{A}}}\left(\mathbf{Z}_{ \pm \mathbf{A}}\right) \rightarrow\left|\mathrm{d}_{L}(\mathbf{A}, \mathbf{r})\right|<\varepsilon_{\mathbf{A}}
\end{aligned}
$$

where $E B_{\delta_{\mathbf{O}}}$ and $E B_{\delta_{\mathbf{A}}}$ are Euclidean balls of respective radii $\delta_{\mathbf{O}}$ and $\delta_{\mathbf{A}}$, centered either in $\mathbf{Z}_{+} \mathbf{A}$ or $\mathbf{Z}_{-\mathbf{A}}$ (see Figure 3).

Let:

$$
E B_{\delta_{M}}\left(\mathbf{Z}_{ \pm \mathbf{A}}\right)=E B_{\delta_{\mathbf{O}}}\left(\mathbf{Z}_{ \pm \mathbf{A}}\right) \cap E B_{\delta_{\mathbf{A}}}\left(\mathbf{Z}_{ \pm \mathbf{A}}\right)
$$

Then, by (2.16), (2.17) and (2.18):

$$
\forall \mathbf{r} \in E B_{\delta_{\mathbf{M}}}\left(\mathbf{Z}_{ \pm \mathbf{A}}\right) \rightarrow\left\{\begin{array}{l}
\left|\mathrm{d}_{L}(\mathbf{O}, \mathbf{r})\right|<\varepsilon_{\mathbf{O}} \\
\left|\mathrm{d}_{L}(\mathbf{A}, \mathbf{r})\right|<\varepsilon_{\mathbf{A}}
\end{array}\right.
$$

The balls $E B_{\delta_{M}}\left(\mathbf{Z}_{ \pm \mathbf{A}}\right)$ are not empty, because each contains at least one of the points $\mathbf{Z}_{ \pm \mathbf{A}}$. Furthermore, because both points $\mathbf{Z}_{ \pm \mathbf{A}}$ are adherence points of the intersection of the interior of both lightcones, every ball centered on any of them contains some point of said interior. Call $\Delta_{\delta_{M}}\left(\mathbf{Z}_{ \pm \mathbf{A}}\right)$ 
the nonempty intersections of $E B_{\delta_{M}}\left(\mathbf{Z}_{ \pm \mathbf{A}}\right)$ with the interior of both cones (see Figure 3):

$$
\Delta_{\delta_{M}}\left(\mathbf{Z}_{ \pm \mathbf{A}}\right)=E B_{\delta_{M}}\left(\mathbf{Z}_{ \pm \mathbf{A}}\right) \cap L^{+}(\mathbf{O}) \cap L^{-}(\mathbf{A}) \neq \Phi
$$

Because every point in $\Delta_{\delta_{M}}\left(\mathbf{Z}_{ \pm \mathbf{A}}\right)$ is also a point of $E B_{\delta_{M}}\left(\mathbf{Z}_{ \pm \mathbf{A}}\right)$, its Lorentzian distance to $\mathbf{O}$ and to $\mathbf{A}$ is (by (2.19)) smaller than $\varepsilon_{\mathbf{O}}$ and $\varepsilon_{\mathbf{A}}$, respectively. These points are therefore contained in both Lorentzian balls $L B_{\varepsilon_{\mathbf{O}}}(\mathbf{O})$ and $L B_{\varepsilon_{\mathbf{A}}}(\mathbf{A})$, which means that they are also contained in the respective arbitrary neighborhoods of $\mathbf{O}$ and $\mathbf{A}$, and consequently, contained in their intersection.

Furthermore, these points also belong to the interior of both conic zones, making them elements of the more severely restricted topological space. This implies that the intersection of these two severely restricted neighborhoods is not empty. Consequently, they are not disjoint.

Since $\mathbf{O}$ and $\mathbf{A}$ are arbitrary, and their selected Lorentzian neighborhoods are arbitrary as well, it follows that no distinct points of the interior of the lightcones have disjoint Lorentzian neighborhoods.

As a conclusion, the subspace so defined is not Hausdorff, even if restricted to the interior of the lightcones.

Figure 3 schematizes a simpler and more intuitive version of this demonstration:

The intersection of the conic-hyperbolic neighborhoods future to $\mathbf{O}$ and past to $\mathbf{A}$ always contains points of $R^{2}$ other than the $Z_{ \pm \mathbf{A}}$, no matter how "thin" these Lorentzian neighborhoods may become.

The above demonstration may be easily extended to $R^{N}$.

\subsubsection{Answering question 2 of $\S 2.2$.}

1. Yes. One-dimensional mathematical analysis may be performed by applying Lorentzian distance functions to the one-dimensional space formed by any spacetime path, with the conditions that this path:

- Be predefined and predetermined, and

- Be contained within the lightcones (excluding the boundary or surface of the cones).

This is so because under these conditions, expression (4) may be replaced by $d \tau=\sqrt{1-\beta^{2}} d t=\lambda d t$ where $\beta$ is the predefined instantaneous speed (of the particle describing the given path) and $0<\lambda \leq$ $1 \in R$. 
2. But, the one-dimensional results obtained by means of the procedure outlined above may not always be linearly combined in order to obtain analytical expressions valid in higher-dimensional spaces. For instance, consider the following scalar field:

$$
F(\mathbf{r})=\left\{\begin{array}{cc}
\frac{x y^{2}}{x^{2}+y^{2}} & x \neq 0 \\
0 & x=0
\end{array} \text { where } \mathbf{r} \in R^{2}, \mathbf{r}=x \hat{\mathbf{i}}+y \hat{\mathbf{j}}\right.
$$

and verify that the derivatives of $F$ at $\mathbf{r}=\mathbf{0}$, along the $\hat{\mathbf{i}}, \hat{\mathbf{j}}$, and $\hat{\mathbf{i}}+\hat{\mathbf{j}}$ vectors, cannot be linearly related ${ }^{17}$

Being able to detemine when a feature is applicable -and when it is not- is as important as the feature itself. (This thought will become an essential part of the present discussion).

3. In order to determine when one-dimensional analytical results may (or may not) be linearly combined into expressions valid in multidimensional spaces, the Euclidean metric (or any of its equivalent metrics) must be used. This will be demonstrated by considering firstly the use of a multidimensional Euclidean metric $\mathrm{d}_{E}{ }^{18}$ and secondly considering the use of other distance functions and their respectively induced topologies.

\section{Linearity in Euclidean topologies:}

Well-known analytical treatises demonstrate that directional derivatives that are continuous throughout an open neighborhood $V_{E}$ of a given point in the domain of a scalar field are also linear with respect

\footnotetext{
${ }^{17}$ Note that $F$ has directional derivatives in every direction at the point $\mathbf{r}=0$, each of which is continuous in its respective topological one-dimensional space. Notwithstanding, these derivatives are not continuous at $r=0$ in a Euclidean $R^{2}$, which is an essential feature for directional derivative linearity, as will be shown.

${ }^{18}$ Because continuity is a topological property, some topology must be defined before making use of this concept in the next paragraph.

Continuity is required for directional derivative linearity because this feature is based on the application of the mean value theorem within a neighborhood converging to the point of analysis. As the neighborhood converges, the directional derivative, applied on the mean value, is required to converge as well.
} 
to the direction of analysis if [46] and only if [47] such scalar field is continuously derivable in such open neighborhood.

\begin{tabular}{|c|c|c|}
\hline $\begin{array}{l}\text { function is } \\
\text { continuously } \\
\text { derivable } \\
\text { (in } V_{E} \text { with } \mathbf{d}_{E} \text { ) }\end{array}$ & $\underbrace{[46]}_{[47]}$ & $\begin{array}{l}\text { directional } \\
\text { derivatives are } \\
\text { linear and } \\
\text { continuous } \\
\text { (in } V_{E} \text { with } \mathrm{d}_{E} \text { ) }\end{array}$ \\
\hline
\end{tabular}

Figure 4: Relation between function derivability and derivative

\section{Testing linearity with Euclidean commensurable metrics:}

Now, if another multidimensional metric $\mathrm{d}_{S}$ is used, that generates a topology stronger (finer) than that generated by the Euclidean metric, then continuity of a given function (in $V_{S}$ with $\mathrm{d}_{S}$ ) does not imply its continuity (in some ${ }^{19} V_{E} \subseteq V_{S}$ with $\mathrm{d}_{E}$ ), so [46] cannot be applied to it.

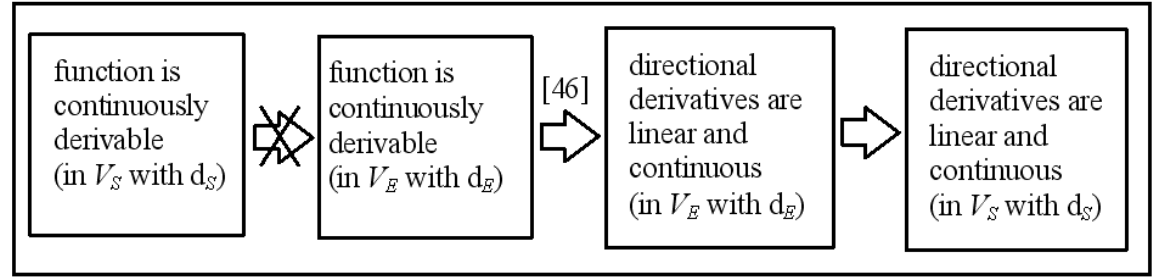

Figure 5: Effect of a stronger topology in the function/derivative relation

\footnotetext{
${ }^{19}$ When shifting from stronger to weaker topologies, there is not only a relaxation of the continuity feature of functions. Because $\mathrm{d}_{S}$ is finer than $\mathrm{d}_{E}, V_{S}$ could well not contain any neighborhoods of the Euclidean topology. Thus, it could happen that no $V_{E} \subseteq V_{S}$.
} 
The inverse implication demonstrated in [47] cannot be applied either:

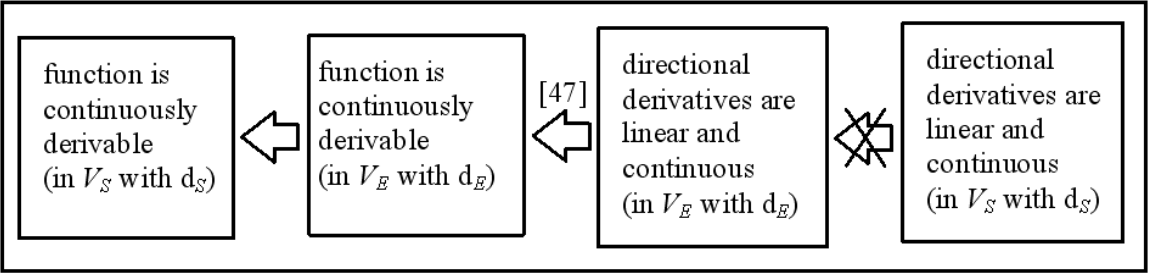

Figure 6: Converse effect of a stronger topology

A similar analysis shows that, if a metric $d_{W}$ is used, that generates a topology weaker (coarser) than that generated by the Euclidean metric then, because continuity of a given function (in $V_{E}$ with $\mathrm{d}_{E}$ ) does not imply its continuity (in some ${ }^{20} V_{W} \subseteq V_{E}$ with $\mathrm{d}_{W}$ ), the results of applying [46] or [47] cannot be characterized in terms of continuity, in the resultant weaker topology:

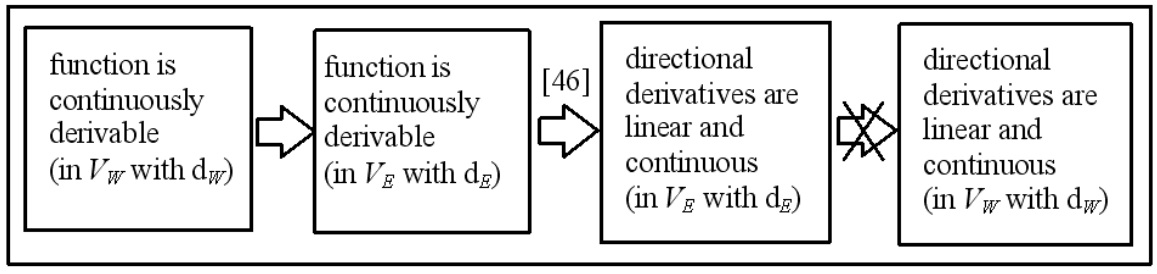

Figure 7: Effect of a weaker topology in the function/derivative relation

\footnotetext{
${ }^{20} \mathrm{As}$ in footnote 19 , because $\mathrm{d}_{W}$ is coarser than $\mathrm{d}_{E}, V_{E}$ could well not contain any neighborhoods of the weaker topology. I.e., it could happen that no $V_{W} \subseteq V_{E}$.
} 


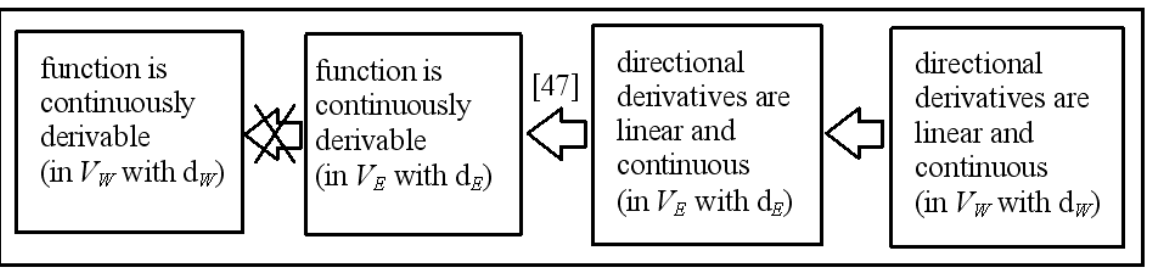

\section{Figure 8: Converse effect of a weaker topology}

Topologies neither stronger nor weaker than that induced by the Euclidean metric allow simultaneous application of [46] and [47].

Testing linearity with Euclidean incommensurable metrics:

If the selected multidimensional metric is incommensurable with the Euclidean metric, then an analysis along this line of thought is inconclusive.

\section{Testing linearity with pseudometrics:}

If a pseudometric is used instead of the Euclidean metric in the multidimensional space, then there is no way to determine when the directional derivatives of a given scalar field are linear. This, because:

- The continuously derivable nature of a scalar field, in an open neighborhood of a given point implies its continuity in said point. This continuity requirement, jointly with the continuity requirement imposed on the directional derivatives (both requirements needed for directional derivative linearity, as shown above), imply that the field (and its directional derivatives) limits must be unique throughout a neighborhood of such a point.

- Guarantee of limit uniqueness implies that the space on which these functions are defined must be Hausdorff, requiring the usage of a proper metric. 
Testing for applicability of the chain rule:

A similar analysis may be done for the application of the chain rule. Consider the Euclidean metric $\mathrm{d}_{E}$ used above. Then:

- The chain rule is applicable at a given point of a continuous path drifting through the domain of a scalar field if such a scalar field is continuously derivable in an open neighborhood $V_{E}$ of said point, in the space that hosts all possible paths [48]. The inverse implication between these features is trivial, considering [46] and [47].

Same as above, the only topology that validates the double implication is that generated by the multidimensional Euclidean metric.

\section{Conclusions}

As a consequence, the topology generated by the Euclidean metric is the only one that enables characterizing the family of functions for which:

- the chain rule may be used

- one-dimensional results may be linearly combined into analytical expressions valid in multidimensional spaces.

Both above-mentioned properties: linearity and the chain rule are required $^{21}$ in order to write the expression for the Minkowski metric:

$$
d s^{2}=g_{\mu \nu} d x^{\mu} d x^{\nu} \text { or more properly } d s^{2}=\eta_{\mu \nu} d x^{\mu} d x^{\nu}
$$

As seen, however, there is no way to identify which functions exhibit the required properties and which do not, when the Lorentzian signature condition is imposed ${ }^{22}$ :

$$
\operatorname{det}\left(g_{\mu \nu}\right)=-1 \text { or more properly } \operatorname{det}\left(\eta_{\mu \nu}\right)=-1
$$

\footnotetext{
${ }^{21}$ Wald [29] and Misner et al. [30], [49] make the explicit assumption that linearity and the chain rule are applicable when deriving (2.21) in their developments.

${ }^{22} R^{4}$ must be assumed in order to use Einstein's way of imposing the Lorentzian signature condition (2.22). Other more general ways of expressing this same condition exist. Nevertheless, whichever way is chosen to impose the signature condition on the metric, it produces the same effect of destroying its positive definiteness.
} 
Consequently, equations (2.21) and (2.22), intended to offer more general expressions for the Lorentzian pseudometrics than (4), are as analytically meaningless as (4).

Similarly, linearity and the chain rule are required, but cannot be guaranteed, when writing STA expressions such as:

$$
\begin{aligned}
& a \cdot \nabla F(x)=\lim _{\varepsilon \rightarrow 0} \frac{F(x+\varepsilon a)-F(x)}{\varepsilon}=a \cdot \gamma^{\mu} \partial_{\mu} F(x) \\
& a \cdot \gamma^{\mu} \partial_{\mu} F(x)=a^{\mu} \partial_{\mu} F(x)=\lim _{\varepsilon \rightarrow 0} \frac{F(x+\varepsilon a)-F(x)}{\varepsilon}
\end{aligned}
$$

where $F$ can be any multivector valued function of spacetime position, $\nabla \equiv \gamma^{\mu} \partial_{\mu}$, and $\left\{\gamma_{\mu} ; \mu=0,1,2,3\right\}$ is a right-handed orthonormal frame of vectors in a Minkowski manifold $M^{4}$ with $\gamma_{0}$ in the forward light-cone, such that $\eta_{\mu \nu}=\gamma_{\mu} \cdot \gamma_{\nu}$.

As a consequence, STA expressions like $d F(x)=d a \cdot \nabla F(x)$ where da is a multivector differential in a Lorentzian topology and $d F(x)$ is a differential of $F$ at $x$, in the space where $F$ is defined, do not guarantee the simultaneous convergence of their left and right hand sides. In fact, $d F(x)$ well could not even be a differential, if defined as above.

\subsubsection{Answering question 3 of $\S 2.2$.}

As indicated, strategies of the type characterized in 3 have been questioned not due to their mathematical merits (or demerits), but due to the fact that they preclude visualizing the Lorentzian topology of spacetime, one of the hallmarks of TSR and TGR.

The first to explore this possibility was Minkowski in 1908 [33]. It is based in a change of variables:

Let $\mathbf{r}$ be a vector in the 4-dimensional spacetime $R^{4}$, and use the following representation:

$$
\mathbf{r}=(c t, \quad x, y, z),
$$

where $t$ stands for time, $c$ for the speed of light in vacuum, and $x, y, z$ for the spatial coordinates ${ }^{23}$.

\footnotetext{
${ }^{23}$ When representing spacetime in vector notation, authors traditionally reserve the lowest-indexed component for representing time. However, when representing (subspaces of) spacetime in graphical way, the axe traditionally indexed as the highest is preferred to represent the temporal dimension.
} 
The following change of coordinates in the $t$ axe:

$$
\mathrm{w}=\sqrt{-1} c t=\mathrm{i} c t
$$

gives the expression of the Lorentz segment differential:

$$
d s^{2}=-c^{2} d t^{2}+d x^{2}+d y^{2}+d z^{2}
$$

an Euclidean appearance in the complex domain:

$$
d s^{2}=d \mathrm{w}^{2}+d x^{2}+d y^{2}+d z^{2}
$$

But, the integral of (2.28) along any null geodesic connecting two different points in the complex domain is still zero. F.i., it is zero along the straight line connecting:

$$
\begin{aligned}
& \mathbf{r}_{0}=\left(\begin{array}{llll}
0, & 0, & 0, & 0
\end{array}\right) \text { and } \\
& \mathbf{r}_{1}=\left(\begin{array}{llll}
\mathrm{i} c \sqrt{x^{2}+y^{2}+z^{2}}, & x, & y, \quad z
\end{array}\right)
\end{aligned}
$$

This means that the Lorentz segment does not constitute a proper metric in the complex domain, just as it did not in the real domain. The topological space formed on $C^{4}$ by this metric is not a Hausdorff space. Convergent sequences are not guaranteed to have unique limits, precluding the perfection of the essential analytical processes.

Discussion: The problem lies in the change of variables (2.26), which does not produce a complete migration of the pseudometric space's underlying set into the field of the complex numbers.

It is a partial migration because inner products in a complex domain $C$ must comply -amongst others- with two axioms. The inner product of two complex elements $\mathrm{x}, \mathrm{y} \in C$ and a scalar $\alpha \in C$ must verify [35]:

$$
\begin{aligned}
\langle\alpha \mathrm{x} \mid \mathrm{y}\rangle & =\alpha\langle\mathrm{x} \mid \mathrm{y}\rangle \\
\langle\mathrm{y} \mid \mathrm{x}\rangle & =\overline{\langle\mathrm{x} \mid \mathrm{y}\rangle}
\end{aligned}
$$

The bar denotes complex conjugate.

The expression for $d s^{2}$ in (2.28), (which should be written $d s^{2}=\langle d s \mid d s\rangle$, the inner product of $d s$ with itself) does not abide by these inner product axioms.

A similar analysis may be done for the STA geometric product definition:

$$
X Y=X \cdot Y+X \wedge Y
$$


(where $X$ and $Y$ are multivectors in a $2^{N}$-dimensional complex space), concluding that it does not abide by these axioms either, neither do none of its product terms: the dot (or inner) product, nor the wedge (or outer) product.

Partial migrations of the pseudometric space's underlying field to complex numbers have also been used for more elaborate purposes, like achieving convergence of sums of histories in quantum mechanics' many-worlds interpretation, and for eliminating singularities, frontiers and associated boundary conditions in cosmology models [50], [51].

\subsubsection{Answering question 4 of $\S 2.2$.}

Subsections $\S 2.3 .1$ and $\S 2.3 .2$ provide a conclusive answer regarding strategy number 4 in $\S 1.34$ :

Copying from $\S 2.3 .1$ :

Since no pseudometric space is a Hausdorff space, sequences are not guaranteed therein to converge to limits that are unique. In these spaces, equals cannot be substituted for equals and therefore the fundamental processes of mathematical analysis cannot be uniquely defined. This invalidates all analytical constructions in the traditional spacetime.

Copying from $\S 2.3 .2$ :

Three theories of convergence are known to-date:

- The theory of sequential convergence, which declares that in pseudometric spaces there is no guarantee of limit uniqueness for convergent sequences.

- The net theory of convergence, which has been proved equivalent to the sequential theory of convergence.

- The filter theory of convergence, which does not endorse limit uniqueness in pseudometric spaces, as this would constitute an essential and extremely conspicuous difference respect the other two theories, giving way for a conclusive negative declaration in respect of its suspected equivalence with the other two theories.

This enables formulating a negative conclusion regarding strategy 4 above. Works such as those detailed in [18] or [34], even Hestenes' projective geometry, offer interesting geometrical concepts, but provide no analytical framework for pseudometric spaces. 


\subsubsection{Triple-check.}

The findings of $\S 2.4 .1$ through $\S 2.4 .4$ above, coherent with the fundamental conclusions under $\S 2.3 .1$ and $\S 2.3 .2$, constitute "personalized" demonstrations of why today's most popular strategies for saving the conflict declared herein are useless.

These individual or "personalized" demonstrations were offered in addition to the general demonstration of $\S 2.3 .1$ considering the generalized adhesion to many of them, and the dramatic impact that this work would bring on them if deemed correct.

In order not to leave way to any doubts regarding the veracity of the claimed statement, a triple-check -from a totally different viewpoint- will be offered: it will be demonstrated -by reductio ad absurdum- that the requirement of continuous derivability for defining gradients or multidimensional derivatives in $R^{N}$ is of the essence of their definition. In effect:

If mathematical analysis could be made possible in a multidimensional pseudometric space by linear combinations of results obtained from onedimensional analyses, irrespective of the nature of the resultant metric, then a way would have been found to circumvent Hausdorff's theorem, which declares pseudometric spaces inapt for these purposes.

This last demonstration by reductio ad absurdum constitutes a triplecheck and supports the verdict coherence and generality announced in $§ 1.4$.

\subsubsection{Answering question 5 in $\S 1.5$.}

The fifth strategy, under 1 of $\S 1.5$ is hereby copied, for easy reference:

1. Looking for another metric that would allow (under the known mathematical theories) building the desired analytical model.

If no constraints are imposed on the resultant model (other than faithfully representing our perceptions of nature), this strategy is perfectly feasible. Other metrics may indeed be selected for mathematical analysis in spacetime.

The essence of the problem, though, is not whether the possibility to choose metrics exists, but how much of the original theories may be retained and how much is to be discarded when employing said chosen metrics.

Fact 3 in $\S 2.1$ is a fundamental point for this strategy, as it identifies those features of TSR and TGR that must be discarded independently 
of the proper metric chosen. Phrased in a positive way, there are certain specific properties that spacetime must exhibit, independently of the proper metric used to generate it, and these are indicated in fact 3 .

A complete reply to the question of what may be retained and what is to be discarded when employing said chosen metrics is beyond the scope of this work. Notwithstanding, a very important aspect of the answer may be clarified immediately: there is no (proper) metric capable of generating the Lorentzian topology traditionally attributed to spacetime.

In other words, any metric apt for mathematical analysis is incompatible with Lorentzian spacetime topology.

This is so because spacetime with any of the Lorentzian topologies is (per $§ 2.3 .3$ not metrizable:

- Hyperbolic neighborhoods do emerge out of the Lorentz pseudometric, so spacetime is clearly pseudometrizable.

- Furthermore, these hyperbolic neighborhoods may be intersected with past and future lightcones to form the family of open sets that defines the desired topology [37].

- But such a space is not a $T_{1}-$ space.

In effect, under such a scenario the set $\{\mathbf{r}\}$ is not closed, because its complement is not open in a multidimensional vector or multivector space with the described topology.

Consequently, the traditional relativistic spacetime is not separable, is not Hausdorff and is not metrizable (impossible to generate with a metric).

This means that, in order to perform mathematical analysis on spacetime, we must renounce to its traditional Lorentzian topology.

\section{EPILOGUE}

\subsection{Conclusions.}

1. There is no way to guarantee that convergent sequences have a unique limit value in TSR -TGR spacetime.

2. Consequently, the possibility of building an analytical framework in the traditional TSR - TGR spacetime is precluded. 
3. A new (proper) metric and its associated topology must be selected for spacetime, and this means that the topology traditionally attributed to it by TSR and TGR must be discarded, jointly with its Lorentzian pseudometrics.

\subsection{Discussion.}

If the surface or boundary of the lightcones is excluded from the scope of the model, then the set $\{\mathbf{r}\}$ is closed, and $\S 2.3 .3$ guarantees the existence of a metric. In this case, it is the discrete metric. With this metric, all functions are continuous but no sequences converge.

Again, this is not a suitable framework for mathematical analysis.

\subsection{Summary.}

In spite of the experimental success of TSR and TGR, an unresolved axiomatic conflict has been highlighted between these physical theories and functional analysis:

While functional analysis rules that analytical expressions must be based on proper metrics, TSR and TGR make intensive use of Lorentzian pseudometrics, which do not guarantee convergence to a unique limit value, (thereby precluding the possibility to establish analytical expressions). This work concludes that TSR's and TGR's non-positive-definite (Lorentzian) pseudometrics and Lorentzian spacetime topology must be inevitably discarded.

\section{REFERENCES}

1. E. F. Taylor and J.A. Wheeler, "Spacetime Physics", 111. W. H. Freeman and Co. (1963).

2. Robert M. Wald, "General Relativity", 61. The University of Chicago Press, Chicago and London, (1984).

3. C. W. Misner et al., "Gravitation", 53. W.H. Freeman and Co., (1973).

4. B. Z. Vulikh, "Introduction to Functional Analysis for Scientists and Technologists", 71. Pergamon Press, (1963).

5. A.W. Naylor and G.R. Sell, "Linear Operator Theory", 47. Holt, Rinehart and Winston, Inc., (1971). 
6. Ibid. [2], 23.

7. Ibid. [3], 51 .

8. Ibid. [2], 60 .

9. R. Tabenski, "Relativity, Special" Encyclopedia of Applied Physics, Vol 16, 375, (1996).

10. Ibid. [4], 77 .

11. Ibid. [5], 70 .

12. Albert Einstein, "Die Grundlage Der Allgemeinen Relativitätstheorie", Spanish translation by Fidel Alsina F. and Damián Canales F. of original paper published in Annalen der Physik 49, 769 (1916). La Relatividad, Emecé Editores, B. Aires, (1950).

13. Ibid. [2], 9, 22-23.

14. Ibid. [3], 324-325.

15. Lars V. Ahlfors \& Leo Sario, "Riemann Surfaces", 124. Princeton University Press, (1960).

16. H.M. Farkas \& I. Kra, "Riemann Surfaces", 198. Springer Verlag (1980).

17. Encyclopaedia Britannica, "Riemannian Geometry," Vol. 19, p.323d, (1967).

18. Shiing-shen Chern "General Relativity and Differential Geometry", in "Some Strangeness in the Proportion, a Centennial Symposium to Celebrate the Achievements of Albert Einstein", 271-280. Addison Wesley publishing Co., (1980).

19. David O. Hestenes, "Spacetime Physics with Geometric Algebra", in American Journal of Physics, 71 (2.7), pp. 691 - 714, (July, 2003).

20. David O. Hestenes, "Journal of Mathematical Analysis and Applications", 24 (2.2), 313 - 325, Academic Press, (1968).

21. Walter Rudin, "Functional Analysis", 372. Mc Graw-Hill, (1973).

22. Frédéric Riesz, "Über Lineare Funktionalgleichungen", Acta Math. 41. pp.71-98, (1917). 
23. S. Banach, "Fundamenta Math. vol 3, pp.133-181, (1922).

24. Ibid. [17], "Mathematics, History of," Vol. 14, p. $1106 \mathrm{~b}$.

25. Ibid. [17], "Operators, Theory of," Vol. 16, p. 988d.

26. Ibid. [40], 67.

27. Ray Redheffer, "Men of Modern Mathematics. A history chart of mathematicians from 1000 to 1900", produced for IBM by the office of Charles Eames (1966).

28. Ibid. [1], 40.

29. Ibid. [2], 14-17.

30. Ibid [3], 208-217, 226-235, 310.

31. Ibid. [3], 311.

32. André Lichnerowicz, "Topics on Space-Times", in "Battelle Rencontres", 1967 Lectures in Mathematics and Physics, 108. W. A. Benjamin. (1968).

33. Ibid. [1], 36-39.

34. Rolf Lingenberg, "Metric Planes and Metric Vector Spaces", 19-23. John Wiley \& Sons (1979).

35. Ibid. [5], 272-276.

36. Ibid [3], 22.

37. Roger Penrose, "Structure of Space-Time", in "Battelle Rencontres", 1967 Lectures in Mathematics and Physics, 121-134. W. A. Benjamin. (1968).

38. Ibid. [2], 59-60.

39. Ibid. [3], 20-21.

40. John L. Kelley, "General Topology", 68. D. Van Norstrand and Co., Inc. (1955).

41. Ibid. [40], 62 . 
42. Roger Penrose, "Techniques of Differential Topology in Relativity", Sect 1. Soc. for Industrial and Applied Mathematics (1972).

43. Ibid. [40], 83.

44. Ibid. [40], 56 .

45. Ibid. [40], 124-130.

46. Tom M. Apostol, "Calculus", 210. Reverté, S.A. (1967).

47. Ibid. [46], $201 \& 271$.

48. Ibid. [46], 215-216

49. Ibid. [3], 47-63, 77, 79-81.

50. Andrei Linde, "Particle Physics and Inflationary Cosmology" Vol. 5. English translation by Marc Damashek, 271-272, 279. Harwood Academic Publishers, (1990).

51. Stephen W. Hawking, "A Brief History of Time. From the Big Bang to Black Holes". Spanish translation by Miguel Ortuño, 178. Editorial Crítica, (1988).

\section{NORBERTO SÁINZ}

Pontificia Universidad Católica de Valparaíso

Facultad de Ingeniería

Escuela de Ingeniería Industrial

Valparaíso

Chile

e-mail : norberto.sainz@ucv.cl 